\title{
ON THE REGULARITY PROPERTIES FOR SOLUTIONS OF THE CAUCHY PROBLEM FOR THE POROUS MEDIA EQUATION
}

\author{
KAZUYA HAYASIDA
}

(Communicated by Walter D. Littman)

\begin{abstract}
We consider the Cauchy problem for the equation $\partial_{t} u=\Delta u^{m}$ in $R^{N} \times(0, T)$. We assume that $1<m<3 N /(3 N-2)$ and the initial data $u_{0}$ is in $C_{0}^{1}\left(R^{N}\right)$ and $u_{0} \geq 0$ in $R^{N}$. Then we prove that the second derivatives of $u^{m}$ with respect to the space-variable are in $L^{2}\left(R^{N} \times(0, T)\right)$.
\end{abstract}

We consider the Cauchy problem for the porous media equation

$$
\begin{cases}\partial_{t} u=\Delta u^{m} & \text { in } R^{N} \times(0, T) \\ u(x, 0)=u_{0}(x) & \text { on } R^{N}\end{cases}
$$

where $m>1$ and $u_{0}(x) \geq 0$. The problem (1.1) has been studied by many authors. For a detailed account of (1.1) we refer to the work of Peletier [7].

We say that $u(x, t)$ is a solution of $(1.1)$, if

$$
\int_{0}^{T} \int_{R^{\wedge}}\left[u(x, t)^{2}+\left|\nabla_{x} u^{m}(x, t)\right|^{2}\right] d x d t<\infty
$$

and

$$
\begin{aligned}
\int_{0}^{T} \int_{R^{*}}\left(u \partial_{t} \phi\right. & \left.-\nabla_{x} u^{m} \cdot \nabla_{x} \phi\right) d x d t \\
& +\int_{R^{*}} u_{0}(x) \phi(x, 0) d x=0
\end{aligned}
$$

for any continuously differentiable function $\phi(x, t)$ with compact support in $R^{N} \times[0, T)$. The existence of such a solution is due to Sabinina [8] under some condition on $u_{0}$.

We are concerned with the regularity of $u$ in (1.1). The Hölder regularity of $u$ was shown by Caffarelli and Friedman [6]. For $N=1$ the precise

Received by the editors September 21, 1987 and, in revised form, June 30, 1988.

1980 Mathematics Subject Classification (1985 Revision). Primary 35K55, 35K65, 35K 15.

Key words and phrases. Porous media, initial data.

This research was partially supported by Grant-in-Aid for Scientific Research, Ministry of Education. 
Hölder exponent with respect to the space-variable was obtained by Aronson [1]. Similar results for the time-variable were studied by di Benedetto [4], when $N \geq 1$. There arises a question whether the derivative $\partial_{t} u$ is a function or not. Concerning this there are results such as Aronson and Bénilan [2], Bénilan [5], where the assumption on $u_{0}$ is very weak. For a function space $A$ the assertion " $\partial_{t} u \in A$ " is alsmot equivalent to " $\partial_{x_{t}} \partial_{x_{j}} u^{m} \in A, 1 \leq i, j \leq N$ ".

According to [5]

$$
\partial_{t} u \in L^{p}\left([\delta, T] \times B_{R}\right)
$$

for any $1<p<1+1 / m, 0<\delta<T$ and $R>0$, where $B_{R}=\left\{x \in R^{N} ;|x|<\right.$ $R\}$. Further if $N=1$ and $m>2$ particularly, $\partial_{t} u \in L^{\infty}\left(\delta, T ; L^{p}\left(B_{R}\right)\right)$ for any $1<p<1+1 /(m-2)$. In this connection we note also the results in [3], [9] and [10].

Our theorem is stated in the following section.

Let us denote by $\left(\right.$, ) and \|\| the inner product and the norm in $L^{2}\left(R^{N}\right)$, respectively. Let us write $G^{T}=R^{N} \times(0, T)$. Our aim is to prove

Theorem. Suppose $1<m<3 N /(3 N-2)$. Let $u_{0}$ be in $C_{0}^{1}\left(R^{N}\right)$, and let $u_{0} \geq 0$ in $R^{N}$. Let $u$ be a solution of (1.1). Then $\partial_{x_{i}} \partial_{x_{j}} u^{m} \in L^{2}\left(G^{T}\right), 1 \leq i, j \leq N$. More precisely, if $u_{0} \leq M$ in $R^{N}$, then for $\alpha=1-1 / m$

$$
\begin{aligned}
\int_{0}^{T}\left\|\partial_{x_{i}} \partial_{x_{j}} u^{m}\right\|^{2} d t \leq & C\left[\left\|u_{0}^{m}\right\|^{2}+\left\|u_{0}^{m(1-\alpha / 2)}\right\|^{2}\right. \\
& \left.+M^{\alpha m}\left\|\nabla u_{0}^{m}\right\|^{2}+\left(u_{0}^{-\alpha m},\left|\nabla u_{0}^{m}\right|^{2}\right)\right],
\end{aligned}
$$

where $C$ depends on $m, N, T$ and not on $u_{0}, M$.

Our method is to derive a uniform energy inequality for each solution of nondegenerate parabolic equations, which are the regular approximation of (1.1) appearing in [6].

We prove our theorem.

For $\eta>0$ let $u^{\eta}(x, t)$ be the solution of

$$
\begin{cases}\partial_{t} u^{\eta}=\Delta\left(u^{\eta}\right)^{m} & \text { in } R^{N} \times(0, \infty) \\ u^{\eta}(x, 0)=u_{0}(x)+\eta & \text { on } R^{N} .\end{cases}
$$

It is known that $\eta \leq u^{\eta} \leq M+\eta$ and $u^{\eta}$ is classical (cf., e.g., [8]). If we set $v^{\eta}=\left(u^{\eta}\right)^{m}$ and $\psi^{\eta}=\left(u_{0}+\eta\right)^{m},(3.1)$ becomes

$$
\begin{cases}\left(v^{\eta}\right)^{-\alpha} \partial_{t} v^{\eta}=m \Delta v^{\eta} & \text { in } R^{N} \times(0, \infty) \\ v^{\eta}(x, 0)=\psi^{\eta}(x) & \text { on } R^{N}\end{cases}
$$


where $\alpha=1-1 / m$. For simplicity we denote $v^{\eta}(x, t)$ and $\psi^{\eta}(x)$ by $v(x, t)$ and $\psi(x)$, respectively. From the proof in Sabinina [8] we easily see that

$$
\begin{aligned}
\| v^{\eta}(\cdot, t)- & \eta^{m} \| \leq C\left(\left\|\psi-\eta^{m}\right\|\right. \\
& \left.+(M+\eta)^{\alpha m / 2}\|\nabla \psi\|\right), \quad 0<t<T,
\end{aligned}
$$

where $C$ depends on $T$ and not on $\psi, \eta$ and $M$.

Let $\zeta \in C_{0}^{2}\left(R^{N}\right)$ and $\zeta \geq 0$ in $R^{N}$. From $\left(3.1^{\prime}\right)$ we have

$$
\frac{1}{2-\alpha}\left(\zeta, \partial_{t} v^{2-\alpha}\right)+m\left(\zeta,\left|\nabla_{x} v\right|^{2}\right)=\frac{m}{2}\left(\Delta \zeta, v^{2}\right) .
$$

Hence

$$
\begin{aligned}
& \int_{0}^{T}\left(\zeta,\left|\nabla_{x} v\right|^{2}\right) d t=\frac{1}{m(2-\alpha)}\left[\left(\zeta, \psi^{2-\alpha}\right)\right. \\
& \left.\quad-\left(\zeta, v(\cdot, T)^{2-\alpha}\right)\right]+\frac{1}{2} \int_{0}^{T}\left(\Delta \zeta, v^{2}\right) d t .
\end{aligned}
$$

Combining this with (3.2) we obtain

$$
\begin{aligned}
\int_{0}^{T}\left(\zeta,\left|\nabla_{x} v\right|^{2}\right) d t \leq C\left[\left(\zeta, \psi^{2-\alpha}\right)+\left\|\psi-\eta^{m}\right\|^{2}\right. \\
\left.+(M+\eta)^{\alpha m}\|\nabla \psi\|^{2}+\eta^{2 m}\right] .
\end{aligned}
$$

Let $w=-\zeta \partial_{x_{j}}^{2} v$ for $1 \leq j \leq N$. By integration by parts we have

$$
\begin{aligned}
& -(\Delta v, w)=\left(\zeta \nabla_{x} \partial_{x_{j}} v, \nabla_{x} \partial_{x_{j}} v\right) \\
& \quad+\left(\partial_{x} \zeta \cdot \nabla_{m} v, \nabla_{x} \partial_{x_{j}} v\right)-\left(\nabla \zeta \cdot \nabla_{x} v, \partial_{x_{j}}^{2} v\right),
\end{aligned}
$$

where we have assumed that $v(\cdot, t) \in C^{3}\left(R^{N}\right)$. But (3.4) is valid for any $v(\cdot, t) \in C^{2}\left(R^{N}\right)$ by taking an approximating sequence of $v$. Similarly we have

$$
\begin{aligned}
\left(v^{-\alpha} \partial_{t} v, w\right)=- & \alpha\left(v^{-\alpha-1} \partial_{x_{j}} v \cdot \partial_{t} v, \zeta \partial_{x_{j}} v\right) \\
& +\left(v^{-\alpha} \partial_{t} \partial_{x_{j}} v, \zeta \partial_{x_{j}} v\right)+\left(v^{-\alpha} \partial_{t} v, \partial_{x_{j}} \zeta \cdot \partial_{x_{j}} v\right) .
\end{aligned}
$$

It is easy to see that

$$
\begin{gathered}
\left(v^{-\alpha-1} \partial_{x_{j}} v \cdot \partial_{t} v, \zeta \partial_{x_{j}} v\right)=m\left(\zeta v^{-1} \Delta v,\left(\partial_{x_{j}} v\right)^{2}\right) \\
\left(v^{-\alpha} \partial_{t} \partial_{x_{j}} v, \zeta \partial_{x_{j}} v\right)=\frac{1}{2}\left(\zeta v^{-\alpha}, \partial_{t}\left(\partial_{x_{j}} v\right)^{2}\right)
\end{gathered}
$$

and

$$
\left(v^{-c} \partial_{t} v, \partial_{x_{j}} \zeta \cdot \partial_{x_{j}} v\right)=m\left(\Delta v, \partial_{x_{j}} \zeta \cdot \partial_{x_{j}} v\right)
$$

Combining these equalities with (3.5), we have

$$
\begin{aligned}
\int_{0}^{T}\left(v^{-c} \partial_{t} v, w\right) d t \geq & -\frac{\alpha m}{2} \int_{0}^{T}\left(\zeta v^{-1} \Delta v,\left(\partial_{x_{j}} v\right)^{2}\right) d t \\
& -\frac{1}{2}\left(\zeta \psi^{-c},\left(\partial_{x_{j}} \psi\right)^{2}\right)+m \int_{0}^{T}\left(\Delta v, \partial_{x_{j}} \zeta \cdot \partial_{x_{j}} v\right) d t
\end{aligned}
$$


From this and (3.4), $\left(3.1^{\prime}\right)$, it follows that

$$
\begin{aligned}
\int_{0}^{T}\left\|\zeta^{1 / 2} \nabla_{x} \partial_{x_{j}} v\right\|^{2} d t \\
\quad \leq \frac{1}{2 m}\left(\zeta \psi^{-\alpha},\left(\partial_{x_{j}} \psi\right)^{2}\right)+\frac{\alpha}{2} \int_{0}^{T}\left(\zeta v^{-1} \Delta v,\left(\partial_{x_{j}} v\right)^{2}\right) d t \\
\quad-\int_{0}^{T}\left(\Delta v, \partial_{x_{j}} \zeta \cdot \partial_{x_{j}} v\right) d t-\int_{0}^{T}\left(\partial_{x_{j}} \zeta \cdot \nabla_{x} v, \nabla_{x} \partial_{x_{j}} v\right) d t \\
\quad+\int_{0}^{T}\left(\nabla \zeta \cdot \nabla_{x} v, \partial_{x_{j}}^{2} v\right) d t
\end{aligned}
$$

Now by integration by parts

$$
\begin{aligned}
\left(\zeta v^{-1} \partial_{x_{j}}^{2} v,\left(\partial_{x_{j}} v\right)^{2}\right)=- & \left(\partial_{x_{j}} \zeta \cdot v^{-1},\left(\partial_{x_{j}} v\right)^{3}\right) \\
& +\left(\zeta v^{-2},\left(\partial_{x_{j}} v\right)^{4}\right)-2\left(\zeta v^{-1} \partial_{x_{j}}^{2} v,\left(\partial_{x_{j}} v\right)^{2}\right)
\end{aligned}
$$

Thus we have

$$
\left(\zeta v^{-1} \partial_{x_{j}}^{2} v,\left(\partial_{x_{j}} v\right)^{2}\right)=\frac{1}{3}\left[\left(\zeta v^{-2},\left(\partial_{x_{j}} v\right)^{4}\right)-\left(\partial_{x_{j}} \zeta \cdot v^{-1},\left(\partial_{x_{j}} v\right)^{3}\right)\right],
$$

so that (3.6) becomes

$$
\begin{aligned}
& \int_{0}^{T}\left\|\zeta^{1 / 2} \nabla_{x} \partial_{x_{j}} v\right\|^{2} d t \\
& \quad \leq \frac{1}{2 m}\left(\zeta \psi^{-\alpha},\left(\partial_{x_{j}} \psi\right)^{2}\right)+\frac{\alpha}{6} \int_{0}^{T}\left(\zeta v^{-2},\left(\partial_{x_{j}} v\right)^{4}\right) d t \\
& \quad-\frac{\alpha}{6} \int_{0}^{T}\left(\partial_{x_{j}} \zeta \cdot v^{-1},\left(\partial_{x_{j}} v\right)^{3}\right) d t+\frac{\alpha}{2} \sum_{i \neq j} \int_{0}^{T}\left(\zeta v^{-1} \partial_{x_{i}}^{2} v,\left(\partial_{x_{j}} v\right)^{2}\right) d t \\
& \quad-\int_{0}^{T}\left(\Delta v, \partial_{x_{j}} \zeta \cdot \partial_{x_{j}} v\right) d t-\int_{0}^{T}\left(\partial_{x_{j}} \zeta \cdot \nabla_{x} v, \nabla_{x^{\prime}} \partial_{x_{j}} v\right) d t \\
& \quad+\int_{0}^{T}\left(\nabla \zeta \cdot \nabla_{x} v, \partial_{x_{j}}^{2} v\right) d t
\end{aligned}
$$

Here we estimate the quantity $\left(\zeta v^{-2},\left(\partial_{x_{j}} v\right)^{4}\right)$. By integration by parts

$$
\begin{aligned}
\left(\zeta v^{-2} \partial_{x_{j}} v,\left(\partial_{x_{j}} v\right)^{3}\right)= & 2\left(\zeta v^{-2} \partial_{x_{j}} v,\left(\partial_{x_{j}} v\right)^{3}\right) \\
& -3\left(\zeta v^{-1} \partial_{x_{j}}^{2} v,\left(\partial_{x_{j}} v\right)^{2}\right)-\left(\partial_{x_{j}} \zeta \cdot v^{-1},\left(\partial_{x_{j}} v\right)^{3}\right) .
\end{aligned}
$$

Hence

$$
\begin{aligned}
& \left(\zeta v^{-2},\left(\partial_{x_{j}} v\right)^{4}\right)=3\left(\zeta v^{-1} \partial_{x_{j}}^{2} v,\left(\partial_{x_{j}} v\right)^{2}\right)+\left(\partial_{x_{j}} \zeta \cdot v^{-1},\left(\partial_{x_{j}} v\right)^{3}\right) \\
& \leq \frac{9}{2}\left(\zeta,\left(\partial_{x_{j}}^{2} v\right)^{2}\right)+\frac{1}{2}\left(\zeta v^{-2},\left(\partial_{x_{j}} v\right)^{4}\right)+\left(\partial_{x_{j}} \zeta \cdot v^{-1},\left(\partial_{x_{j}} v\right)^{3}\right),
\end{aligned}
$$

which implies that

$$
\left(\zeta v^{-2},\left(\partial_{x_{j}} v\right)^{4}\right) \leq 9\left(\zeta,\left(\partial_{x_{j}}^{2} v\right)^{2}\right)+2\left(\partial_{x_{j}} \zeta \cdot v^{-1},\left(\partial_{x_{j}} v\right)^{3}\right) .
$$


Since $2\left(\partial_{x_{j}} \zeta \cdot v^{-1},\left(\partial_{x_{j}} v\right)^{3}\right) \leq \varepsilon\left(\zeta v^{-1},\left(\partial_{x_{j}} v\right)^{4}\right)+\varepsilon^{-1}\left(\zeta^{-1}\left(\partial_{x_{j}} \zeta\right)^{2},\left(\partial_{x_{j}} v\right)^{2}\right)$ for sufficiently small $\varepsilon>0$, we obtain

$$
\left(\zeta v^{-2},\left(\partial_{x_{j}} v\right)^{4}\right) \leq \frac{9}{1-\varepsilon}\left(\zeta,\left(\partial_{x_{j}}^{2} v\right)^{2}\right)+C(\varepsilon)\left(\zeta^{-1}\left(\partial_{x_{j}} \zeta\right)^{2},\left(\partial_{x_{j}} v\right)^{2}\right)
$$

From this and Cauchy's inequality it follows that

$$
\begin{aligned}
\left|\left(\partial_{x_{j}} \zeta \cdot v^{-1},\left(\partial_{x_{j}} v\right)^{3}\right)\right| \leq & \frac{9 \varepsilon}{2(1-\varepsilon)}\left(\zeta,\left(\partial_{x_{j}}^{2} v\right)^{2}\right) \\
& +C^{\prime}(\varepsilon)\left(\zeta^{-1}\left(\partial_{x_{j}} \zeta\right)^{2},\left(\partial_{x_{j}} v\right)^{2}\right)
\end{aligned}
$$

Now we define

$$
\begin{aligned}
& I_{j}=\frac{\alpha}{6}\left(\zeta v^{-2},\left(\partial_{x_{j}} v\right)^{4}\right)-\frac{\alpha}{6}\left(\partial_{x_{j}} \zeta \cdot v^{-1},\left(\partial_{x_{j}} v\right)^{3}\right) \\
& +\frac{\alpha}{2} \sum_{i \neq j}\left(\zeta v^{-1} \partial_{x_{i}}^{2} v,\left(\partial_{x_{j}} v\right)^{2}\right), \quad 1 \leq j \leq N .
\end{aligned}
$$

Using (3.9) and Cauchy's inequality we have for $\delta>0$

$$
\begin{aligned}
I_{j} & \leq \frac{\alpha}{4 \delta}\left(\zeta,\left(\sum_{i \neq j} \partial_{x_{i}}^{2} v\right)^{2}\right)+\left(\frac{\alpha \delta}{4}+\frac{\alpha}{6}\right)\left(\zeta v^{-2},\left(\partial_{x_{j}} v\right)^{4}\right) \\
& +\frac{3 \alpha \varepsilon}{4(1-\varepsilon)}\left(\zeta,\left(\partial_{x_{j}}^{2} v\right)^{2}\right)+C\left(\zeta^{-1}\left(\partial_{x_{j}} \zeta\right)^{2},\left(\partial_{x_{j}} v\right)^{2}\right) .
\end{aligned}
$$

Easily, $\left(\sum_{i \neq j} \partial_{x_{i}}^{2} v\right)^{2} \leq(N-1) \sum_{i \neq j}\left(\partial_{x_{i}}^{2} v\right)^{2}$. Thus by (3.8) it follows that

$$
\begin{aligned}
\sum_{j} I_{j} & \leq\left[\frac{\alpha}{4 \delta}(N-1)^{2}+\frac{9}{1-\varepsilon}\left(\frac{\alpha \delta}{4}+\frac{\alpha}{6}\right)+\frac{3 \alpha \varepsilon}{4(1-\varepsilon)}\right]\left(\zeta, \sum_{j}\left(\partial_{x_{j}}^{2} v\right)^{2}\right) \\
& +C \sum_{j}\left(\zeta^{-1}\left(\partial_{x_{j}} \zeta\right)^{2},\left(\partial_{x_{j}} v\right)^{2}\right) .
\end{aligned}
$$

Here we put $\delta=(N-1) / 3$. Then

$$
\frac{\alpha}{4 \delta}(N-1)^{2}+9\left(\frac{\alpha \delta}{4}+\frac{\alpha}{6}\right)=3 \alpha N / 2 .
$$

From our assumption on $m$ we see that $3 \alpha N / 2<1$.

Next we estimate the remaining terms on the right-hand side of (3.7). For sufficiently small $\varepsilon^{\prime}>0$

$$
\begin{gathered}
\sum_{j}\left|\left(\Delta v, \partial_{x_{j}} \zeta \cdot \partial_{x_{j}} v\right)+\left(\partial_{x_{j}} \zeta \cdot \nabla_{x} v, \nabla_{x} \partial_{x_{j}} v\right)-\left(\nabla \zeta \cdot \nabla_{x} v, \partial_{x_{j}}^{2} v\right)\right| \\
\leq \varepsilon^{\prime} \sum_{j}\left\|\zeta^{1 / 2} \nabla_{x} \partial_{x_{j}} v\right\|^{2}+C\left(\varepsilon^{\prime}\right)\left(\zeta^{-1}|\nabla \zeta|^{2},\left|\nabla_{x} v\right|^{2}\right) .
\end{gathered}
$$

Therefore combining (3.7), (3.10) with the above, we conclude that

$$
\begin{aligned}
\sum_{j} \int_{0}^{T}\left\|\zeta^{1 / 2} \nabla_{x} \partial_{x_{j}} v\right\|^{2} d t \leq & C\left[\left(\zeta \psi^{-\alpha},|\nabla \psi|^{2}\right)\right. \\
& \left.+\int_{0}^{T}\left(\zeta^{-1}|\nabla \zeta|^{2},\left|\nabla_{x} v\right|^{2}\right) d t\right]
\end{aligned}
$$


Let $\xi(x)$ be in $C_{0}^{\infty}\left(R^{N}\right)$, and let us put $\zeta=\xi^{2}$. Then from the above inequality and (3.3) it follows that for $1 \leq i, j \leq N$

$$
\begin{aligned}
\int_{0}^{T} & \left\|\zeta^{1 / 2} \partial_{x_{i}} \partial_{x_{j}} v^{\eta}\right\|^{2} d t \\
\quad \leq & C\left[\left(\zeta\left(\psi^{\eta}\right)^{-\alpha},\left|\nabla \psi^{\eta}\right|^{2}\right)\right. \\
& \left.+\left(|\nabla \xi|^{2},\left(\psi^{\eta}\right)^{2-\alpha}\right)+\left\|\psi^{\eta}-\eta^{m}\right\|^{2}+(M+\eta)^{\alpha m}\left\|\nabla \psi^{\eta}\right\|^{2}+\eta^{2 m}\right] .
\end{aligned}
$$

As is well known, $v^{\eta} \downarrow v(\eta \downarrow 0)$ in $G$, where $v=u^{m}$ and $u$ is the solution of (1.1). For each positive integer $n$ we put $\xi(x)=\xi_{n}(x)$, where

$$
\xi_{n}(x)= \begin{cases}1 & (|x| \leq n) \\ 0 & (|x|>2 n)\end{cases}
$$

and they are uniformly bounded in $R^{N}$ up to the third derivatives. Then the constant $C$ on the right-hand side of (3.11) are independent of $n$. Letting $\eta \rightarrow 0$ in (3.11), we see that $\partial_{x_{i}} \partial_{x_{j}} v^{\eta} \rightarrow \partial_{x_{i}} \partial_{x_{j}} v$ weakly in $L_{\text {loc }}^{2}(G)$ and $\int_{0}^{T}\left\|\zeta^{1 / 2} \partial_{x_{i}} \partial_{x_{j}} v\right\|^{2} d t \leq$ the right-hand side of (2.1). This completes the proof.

\section{REFERENCES}

1. D. G. Aronson, Regularity properties of flow through porous media, SIAM J. Appl. Math. 17 (1969), 461-467.

2. D. G. Aronson and P. Bénilan, Régularité des solutions de l'équation des mileux poreux dans $R^{N}$, C. R. Acad. Sci. Paris. Sér. A 288 (1979), 103-105.

3. S. Benachour and M. S. Moulay, Régularité des solutions de l'équation des mileux poreux en une dimension d'espace, C. R. Acad. Sci. Paris, Sér. I 298 (1984), 107-110.

4. E. di Benedetto, Regularity results for the porous media equation, Ann. Mat. Pura Appl. (4) 121 (1979), 249-262.

5. P. Bénilan, A strong regularity $L^{p}$ for solution of the porous media equation, Res. Notes in Math. 89, 39-58.

6. L. A. Caffarelli and A. Friedman, Regularity of the free boundary of a gas flow in a ndimensional porous medium, Indiana Univ. Math. J. 29 (1980), 361-391.

7. L. A. Peletier, The porous media equation, in Application of nonlinear analysis in the physical sciences (H. Amman et al., editors), Pitman, New York, 1981, pp. 229-241.

8. E. S. Sabinina, On the Cauchy problem for the equation of nonstationary gas filtration in several space variables, Soviet Math. 2 (1961), 166-169.

9. F. Simondon, Effect régularisant local pour $u_{t}=(\varphi(u))_{x x}$, C. R. Acad. Sci. Paris, Sér. I 299 (1984), 969-972.

10. N. I. Wolanski, A diffiusion problem with a measure as initial datum, J. Math. Anal. Appl. 102 (1984), 365-384.

Department of Mathematics, Faculty of Science, Kanazawa University, Kanazawa 920, JAPAN 